\title{
Transport of Corilagin, Gallic Acid, and Ellagic Acid from Fructus Phyllanthi Tannin Fraction in Caco-2 Cell Monolayers
}

\author{
Xin Mao, ${ }^{1,2}$ Ling-Fang Wu, ${ }^{1}$ Hai-juan Zhao, ${ }^{1}$ Wen-Yi Liang, ${ }^{1}$ Wen-Jing Chen, ${ }^{1}$ \\ Shu-Xian Han, ${ }^{2}$ Qi Qi, ${ }^{1}$ Ya-Ping Cui, ${ }^{1}$ Shi Li, ${ }^{1}$ Guang-Hui Yang, ${ }^{1}$ Yan-Yan Shao, \\ Dan Zhu, ${ }^{1}$ Ru-Feng Wang, ${ }^{1}$ Yun You, ${ }^{2,3}$ and Lan-Zhen Zhang ${ }^{1}$ \\ ${ }^{1}$ School of Chinese Materia Medica, Beijing University of Chinese Medicine, Beijing 100102, China \\ ${ }^{2}$ Institute of Chinese Materia Medica, China Academy of Chinese Medical Sciences, Beijing 100700, China \\ ${ }^{3}$ Key Laboratory of Chinese Internal Medicine, Beijing University of Chinese Medicine, Beijing 100700, China \\ Correspondence should be addressed to Yun You; youyunrice@126.com and Lan-Zhen Zhang; zhanglanzhen01@126.com
}

Received 20 April 2016; Accepted 27 July 2016

Academic Editor: José L. Ríos

Copyright (C) 2016 Xin Mao et al. This is an open access article distributed under the Creative Commons Attribution License, which permits unrestricted use, distribution, and reproduction in any medium, provided the original work is properly cited.

Objective. To investigate the absorption property of the representative hydrolyzable tannin, namely corilagin, and its hydrolysates gallic acid (GA) and ellagic acid (EA) from the Fructus Phyllanthi tannin fraction (PTF) in vitro. Methods. Caco-2 cells monolayer model was established. Influences of PTF on Caco-2 cells viability were detected with MTT assay. The transport across monolayers was examined for different time points, concentrations, and secretory directions. The inhibitors of P-glycoprotein (P-gp), multidrug resistance proteins (MRPs), organic anion transporting polypeptide (OATP) and sodium/glucose cotransporter 1 (SGLT1), and tight junction modulators were used to study the transport mechanism. LC-MS method was employed to quantify the absorption concentration. Results. The apparent permeability coefficient $\left(P_{\text {app }}\right)$ values of the three compounds were below $1.0 \times 10^{-6} \mathrm{~cm} / \mathrm{s}$. The absorption of corilagin and GA were much lower than their efflux, and the uptake of both compounds was increased in the presence of inhibitors of P-gp and MRPs. The absorption of EA was decreased in the company of OATP and SGLT1 inhibitors. Moreover, the transport of corilagin, GA, and EA was enhanced by tight junction modulators. Conclusion. These observations indicated that the three compounds in PTF were transported via passive diffusion combined with protein mediated transport. P-gp and MRPs might get involved in the transport of corilagin and GA. The absorption of EA could be attributed to OATP and SGLT1 protein.

\section{Introduction}

Hydrolyzable tannins belong to the family of polyphenol, which integrated in some kinds of foods and medicinal herbs, such as Punica granatum and Terminalia chebula Retz. Recently, researches of hydrolyzable tannins are getting more and more attentions on account of their benefits for our health [1]. As a typical hydrolyzable tannin, corilagin is a potential antitumor compound $[2,3]$. In addition, it exhibits antiviral, antihyperalgesic, and antibacterial activities [4-6]. Gallic acid (GA) and ellagic acid (EA) are two hydrolysates of corilagin, both of which possess strong antioxidant and anticancer effects [7-10].

Phyllanthus emblica L. (Euphorbiaceae) is a famous medicinal plant distributed in Asia countries especially in
China. Its fruit, Fructus Phyllanthi, has been used for the treatment of liver and digestive diseases in China for a long history $[11,12]$. The recent studies of our team demonstrated that Fructus Phyllanthi tannin fraction (PTF) inhibits the migration and invasion of human lung squamous carcinoma cell line (NCI-H1703) in vitro through MAPK/MMP pathways [13]. Corilagin, EA, and GA are the three main compounds, which are contained about $10.26 \%$ in PTF [14] and the biological effects of PTF are considered to be correlated with these compounds. In order to elucidate the absorption of PTF, an in vitro Caco-2 monolayer model was established.

Caco-2 cells were derived from human colorectal carcinoma, and Caco-2 cell monolayer is the classical cell model of intestinal epithelium $[15,16]$. There are various transporters expressed in the membranes such as P-gp, 
MRPs, OATP, SGLT1, monocarboxylic acid transporters (MCT), and breast cancer resistance protein (BCRP) [1720]. Intestinal absorption of polyphenol compounds, most of which were flavonoids, across Caco- 2 cell monolayer was reported by various researchers [21-23]. These flavonoids such as apigenin, orientin, and (-)epicatechin-3-gallate were hardly transported through the monolayer and possibly via paracellular pathway, and some of them were suggested to be mediated by carrier protein [21-23].

Our previous studies found that the absorption of corilagin, EA, and GA was very poor with peak concentration values $\left(C_{\max }\right)$ lower than $10.47 \mu \mathrm{g} / \mathrm{mL}$ after oral administration of PTF at dose of $6 \mathrm{~g} / \mathrm{kg}$ in rats [14]. It is essential to find out why the low absorption of oral PTF occurred and to identify suitable pharmaceutical methods to improve the absorption of PTF and further enhance the anticancer activities. P-gp inhibitor (verapamil) [21, 22] and MRPs inhibitor (indomethacin) [24, 25] were employed to investigate whether the transport was affected by the efflux transporters, OATP inhibitor (indomethacin) $[26,27]$ and SGLT1 inhibitor (phloridzin) $[21,28]$ were chosen to study the effect of absorption transporters, and tight junction modulators (sodium dodecyl sulfate (SDS) and poly-l-lysine $(\mathrm{PLL}))[29,30]$ were used to evaluate the role of paracellular permeability in the transport of these three compounds from PTF.

\section{Materials and Methods}

2.1. Materials. Corilagin, GA, EA, and fluorescein sodium were purchased from National Institute for the Control of Pharmaceutical and Biological Products (Beijing, China). Phloridzin, indomethacin, verapamil, SDS, and type I collagen (from rat tail) were obtained from Sigma Chemical Co. (St. Louis, MO, USA). PLL (MW: 70,000-150,000) was purchased from ScienCell (Carlsbad, CA, USA). Dulbecco's modified Eagle's minimal essential medium (DMEM) and penicillin-streptomycin were from Thermo (Beijing, China). Fetal bovine serum (FBS), nonessential amino acid (NEAA), and Hank's balanced salt solution (HBSS) came from Gibco Life Technologies (Grand Island, NY, USA). 12well Transwell-Clear plates (pore size $0.4 \mu \mathrm{m}$, surface area $1.12 \mathrm{~cm}^{2}$ ) and 96 -well plates were from Corning Incorporated.

2.2. Extraction and Purification of PTF. The fruit of Phyllanthus emblica L. (Fructus Phyllanthi) grown in Nepal was purchased from the Beijing Tibetan Hospital. The powder of Fructus Phyllanthi was extracted three times through reflux at $60^{\circ} \mathrm{C}$ with ethanol/water $(3: 2 ; \mathrm{v} / \mathrm{v})$, and the extract was combined and concentrated with rotary evaporator (EYELA, NN Series; Rikakikai Co. Ltd., Tokyo, Japan) at $40^{\circ} \mathrm{C}$. The supernatant of condensed liquid was collected by centrifugation and enriched by gradient elution on macroporous resin column. The content of total tannins of PTF determined by tungsten molybdophosphate-casein colorimetric method was higher than 56\%. The HPLC fingerprint of the PTF was studied, from which three main peaks were identified as GA, corilagin, and EA [31]. They were quantitatively analyzed using RP-HPLC method, and their concentrations were $4.72 \% \pm 0.06 \%, 1.87 \% \pm 0.05 \%$, and $3.67 \% \pm 0.04 \%$, respectively [14]. The PTF was dissolved and diluted with HBSS solution to a final concentration.

2.3. Cell Culture. Caco-2 cell line was derived from American Type Culture Collection (Manassas, VA, USA). Caco-2 cells were grown in DMEM, supplemented with $10 \%$ FBS, $1 \mathrm{mM}$ NEAA, $100 \mathrm{U} / \mathrm{mL}$ penicillin, and $0.1 \mu \mathrm{g} / \mathrm{mL}$ streptomycin and cultured in a humidified atmosphere of $5 \% \mathrm{CO}_{2}$ in air at $37^{\circ} \mathrm{C}$.

The type I collagen dissolved in $0.1 \mathrm{M}$ acetic acid was used to coat Transwell-Clear plates at $8 \mu \mathrm{g} / \mathrm{cm}^{2}$; the supernatant was removed after binding for 2 hours at $37^{\circ} \mathrm{C}$. The plates were washed twice with phosphate buffered saline and dried overnight.

When cells were cultured to $80 \%$ confluence, they were seeded in 12-well Transwell-Clear plates at a density of 1.2 $\times 10^{5}$ cells/insert. Cells were cultured for 21 days to reach confluence and functional differentiation. Complete medium was replaced every 2 days for the first 2 weeks and daily renewed for the last 7 days. All cells were between passages 35 and 55. Transepithelial electrical resistance (TEER) values were tested using Millicell ERS-2 Volt-Ohm Meter (Millipore, Billerica, MA, USA), and the TEER values $>500 \Omega / \mathrm{cm}^{2}$ were required.

2.4. Cell Viability Evaluation. MTT assay was employed to test the influence on cell viability of PTF on Caco-2 cells. Caco- 2 cells were seeded in 96-well plate at a density of $2 \times 10^{4}$ cells/well and cultured until confluence. Then, the DMEM culture medium was replaced with $150 \mu \mathrm{L}$ HBSS and incubated with different concentrations of fractions for $4 \mathrm{~h}$. The concentration of PTF was ranged from 0.25 to $2 \mathrm{mg} / \mathrm{mL}$. Thereafter, the HBSS was removed and the monolayers were incubated with $200 \mu \mathrm{L}$ of DMEM culture medium containing MTT $500 \mu \mathrm{g} / \mathrm{L}$ for $4 \mathrm{~h}$. Finally, the medium was removed, the formazan salt crystals that remained were dissolved with $150 \mu \mathrm{L}$ of DMSO and shaken for $10 \mathrm{~min}$, and the absorbance was measured at $570 \mathrm{~nm}$ using microplate reader (Molecular Devices, Sunnyvale, CA, USA) [28]. The cell inhibitory rate was calculated as follows: inhibitory rate $(\%)=\left(\mathrm{A} 570_{\text {control }}-\right.$ $\left.\mathrm{A} 570_{\text {sample }}\right) /\left(\mathrm{A} 570_{\text {control }}-\mathrm{A} 570_{\text {blank }}\right) \times 100 \%$.

2.5. Transport Experiments. After 21-day culture, the complete medium was removed and the monolayer was washed three times and balanced with HBSS (previously warmed to $\left.37^{\circ} \mathrm{C}\right)$ for $30 \mathrm{~min}$. PTF $(0.25,0.50$, and $1.00 \mathrm{mg} / \mathrm{mL})$ was added onto the Apical side $(0.5 \mathrm{~mL})$ or Basolateral side $(1.5 \mathrm{~mL})$, while blank HBSS was added onto the receiving chamber. Aliquots of $200 \mu \mathrm{L}$ samples were taken from the receiving chamber every $30 \mathrm{~min}$ for $180 \mathrm{~min}$ and each time an equal volume of blank HBSS was replenished. All samples were dried with organomation. Before analyzing, the dry samples were dissolved in methanol/water/acetic acid (200:200:1, $\mathrm{v} / \mathrm{v} / \mathrm{v}$ ) and the insoluble substance was eliminated with centrifugation $12000 \mathrm{rpm}$ for $5 \mathrm{~min}$.

Protein inhibitors including verapamil $(100 \mu \mathrm{M})$, indomethacin $(10 \mu \mathrm{M})$, and phloridzin $(50 \mu \mathrm{M})$ were employed to investigate the role of different transporters. Epithelial tight junction modulators such as SDS $(50 \mu \mathrm{g} / \mathrm{mL})$ 
and PLL $(50 \mu \mathrm{g} / \mathrm{mL})$ were used to evaluate the role of paracellular permeability.

Hydrophilic marker sodium fluorescein $(0.6 \mathrm{mg} / \mathrm{mL})$ was used for the evaluation of the integrity of the cell monolayer. The absorbance of the marker was measured at $490 \mathrm{~nm}$ using the microplate reader (Molecular Devices, Sunnyvale, CA, USA), and the concentration of sodium fluorescein was calculated by standard curve. The $P_{\text {app }}$ for the hydrophilic marker was required lower than $1.00 \times 10^{-6} \mathrm{~cm} / \mathrm{s}$.

2.6. LC-MS Analysis. The analytes were analyzed on a Diamonsil C18 column $(250 \times 4.6 \mathrm{~mm}, 5 \mu \mathrm{m}$ i.d., Dikma Technologies Inc., CA, USA) using an Agilent 1260 series liquid chromatograph (Agilent Technologies, CA, USA) comprised of an auto injector, a quaternary pump, a diode array detector (DAD), and a column counterpart. The elution was performed with mobile phase composed of $0.1 \%$ formic acid in acetonitrile (solvent $\mathrm{A}$ ) and $0.1 \%$ formic acid in water (solvent $\mathrm{B}$ ) using the following gradient program at the flow rate of $1 \mathrm{~mL} / \mathrm{min}: 0-12 \mathrm{~min}$, linear gradient $15-65 \%(\mathrm{~A}) ; 12-$ $13 \mathrm{~min}$, linear gradient $65-100 \%$ (A); $13-25 \mathrm{~min}$, isocratic gradient $100-100 \%$ (A). The column temperature was $20^{\circ} \mathrm{C}$ and the injection volume was $20 \mu \mathrm{L}$.

MS/MS analysis was acquired using an Agilent 6410 triple quadrupole mass spectrometer (Agilent Technologies, CA, USA) equipped with an electrospray ionisation (ESI) in the negative mode. Other analytical conditions included the drying gas temperature of $300^{\circ} \mathrm{C}$, the drying gas flow of $11 \mathrm{~L} / \mathrm{min}$, the nebulizer pressure of $35 \mathrm{psi}$, and the capillary voltage of $4 \mathrm{kV}$. To get the highest abundance, the fragmentor voltage and collision energy were optimized. Data acquisition and processing were accepted by Agilent Mass Hunter workstation software version B.05.00 using multiple reaction monitoring (MRM). The quantification was performed using external standard method.

2.7. Data Analysis. The $P_{\text {app }}$ and PDR were determined according to the equation:

$$
\begin{aligned}
P_{\text {app }} & =\frac{(\mathrm{d} C / \mathrm{d} t \times V)}{\left(A \times C_{0}\right)}, \\
\mathrm{PDR} & =\frac{P_{\text {app }(\mathrm{BL} \rightarrow \mathrm{AP})}}{P_{\mathrm{app}(\mathrm{AP} \rightarrow \mathrm{BL})}}
\end{aligned}
$$

Here, $\mathrm{d} C / \mathrm{d} t$ is the change in concentration on the receiver side over time; $V$ is the volume of the solution in the receiver chamber; $A$ is the surface area of the cell monolayer; $C_{0}$ is the initial concentration in the donor chamber. PDR is the ratio of $P_{\text {app }(\mathrm{BL} \rightarrow \mathrm{AP})}$ to $P_{\text {app }(\mathrm{AP} \rightarrow \mathrm{BL})}$.

The results presented in this study were the averages of at least three replicates and were expressed as the mean \pm SEM. Differences amongst treatments were detected by analysis of one-way ANOVA. The differences were considered to be significant when $P<0.05$.

\section{Results}

3.1. Validation of the Caco-2 Monolayers. The TEER values of the monolayers increased over the first 12 days and steadily

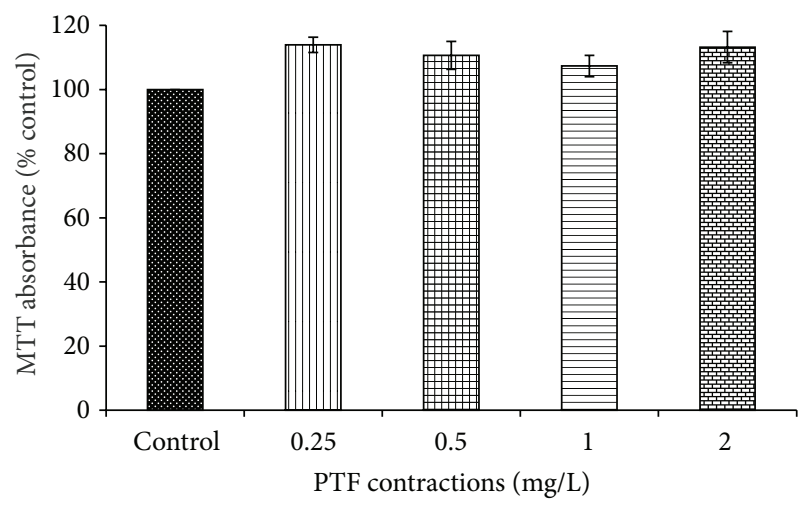

FIGURE 1: Effects of PTF on viability of Caco-2 cells. Results are expressed as the mean \pm SEM for 10 determinations.

for the last 9 days. The values were above $500 \Omega / \mathrm{cm}^{2}$ on day 21. The $P_{\text {app }}$ value of sodium fluorescein, which is a poorly transported marker, tested across the monolayers was $3.16 \pm$ $0.25 \times 10^{-7} \mathrm{~cm} / \mathrm{s}$ which agreed with that published in the previous literature $[32,33]$.

3.2. Cell Viability Evaluation. At the concentrations of 0.25 , $0.50,1.00$, and $2.00 \mathrm{mg} / \mathrm{mL}$, no significant effects on growth of Caco- 2 cells were observed in $4 \mathrm{~h}$ (Figure 1), which indicated that PTF exhibited no toxic to Caco-2 cells at 0.25$2.00 \mathrm{mg} / \mathrm{mL}$ for $4 \mathrm{~h}$.

3.3. Quantification of Analytes in Herbal Extracts by LC-MS. The MS conditions of LC-MS method had been optimized to achieve the highest abundance of corilagin, GA, and EA when the MRM mode was used and the $[M-1]^{-}$was employed as the precursor ion (Figure 2). For corilagin the retention time was $6.61 \mathrm{~min}$, the mass transition used was $\mathrm{m} / \mathrm{z}$ $633.1 \rightarrow 301.0$ (collision energy, $-35 \mathrm{eV}$ ), the linear equation was $Y=23.705 X-26.106\left(r^{2}=0.9999\right)$ with a good linearity over the range from $0.65 \mathrm{ng} / \mathrm{mL}$ to $653 \mathrm{ng} / \mathrm{mL}$, the limit of detection (LOD, signal-to-noise ratio $\geq 3$ ) was $1.35 \mathrm{ng} / \mathrm{mL}$, and the limit of quantitation (LOQ, signal-to-noise ratio $\geq 10$ ) was $1.94 \mathrm{ng} / \mathrm{mL}$. For GA the retention time was $4.14 \mathrm{~min}$, the mass transition used was $m / z 169.0 \rightarrow 125.0$ (collision energy, $-10 \mathrm{eV})$, the linear equation was $Y=28.769 \mathrm{X}+22.697\left(r^{2}=\right.$ 0.9999 ) with a good linearity over the range from $1.01 \mathrm{ng} / \mathrm{mL}$ to $252 \mathrm{ng} / \mathrm{mL}$, the LOD was $0.08 \mathrm{ng} / \mathrm{mL}$, and the LOQ was $1.64 \mathrm{ng} / \mathrm{mL}$. For EA the retention time was $8.39 \mathrm{~min}$, the mass transition used was $\mathrm{m} / z 301.0 \rightarrow 301.0$ (collision energy, $-10 \mathrm{eV})$, the linear equation was $Y=111.21 \mathrm{X}+130.99\left(r^{2}=\right.$ 0.9982 ) with a good linearity over the range from $1.98 \mathrm{ng} / \mathrm{mL}$ to $989 \mathrm{ng} / \mathrm{mL}$, the LOD was $0.17 \mathrm{ng} / \mathrm{mL}$, and the LOQ was $0.97 \mathrm{ng} / \mathrm{mL}$.

Three concentrations of corilagin, GA, and EA in six replicates in a single day were analyzed as intraday precision and duplicating the intraday experiment on two successive days was analyzed as interday precision. The precision and recovery data are shown in Table 1. 


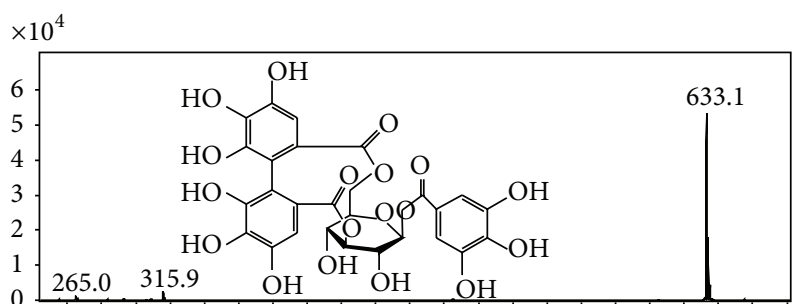

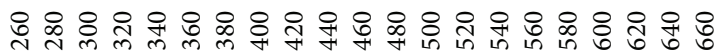

$(\mathrm{m} / z)$

(a1)

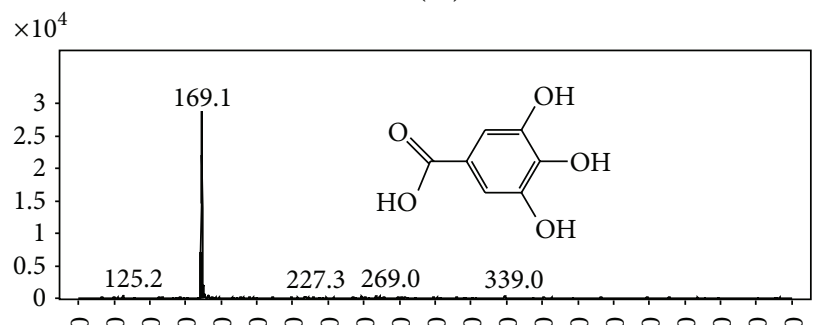

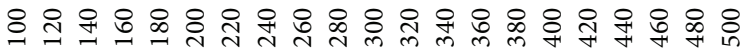

$(m / z)$

(b1)

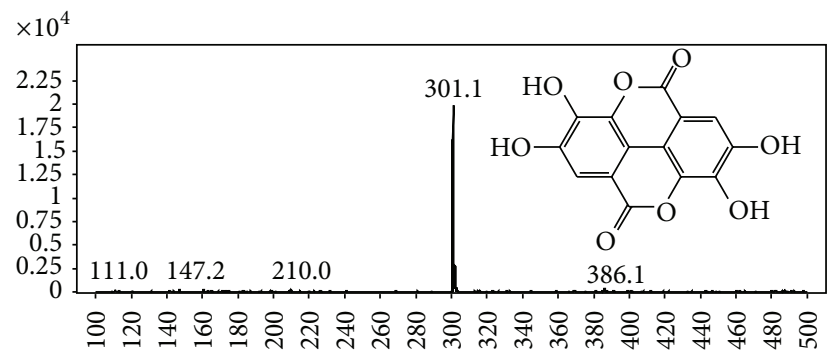

$(\mathrm{m} / z)$

(c1)

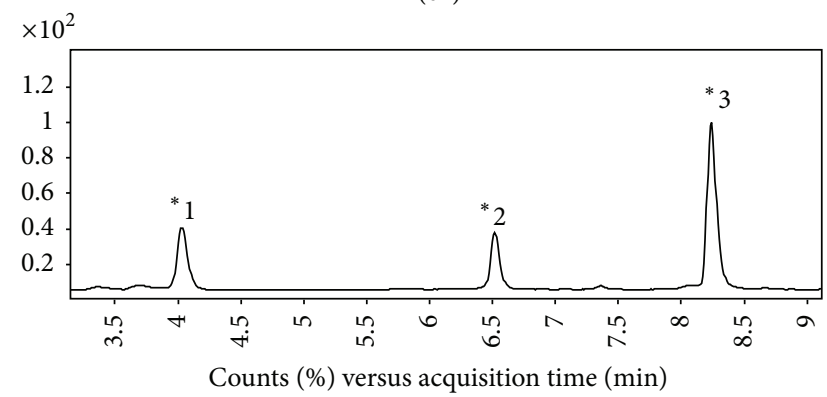

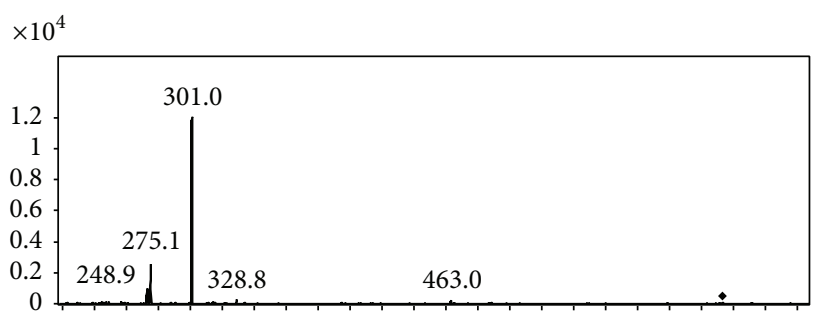

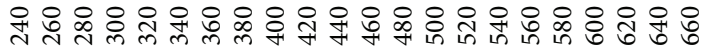

$(\mathrm{m} / z)$

(a2)

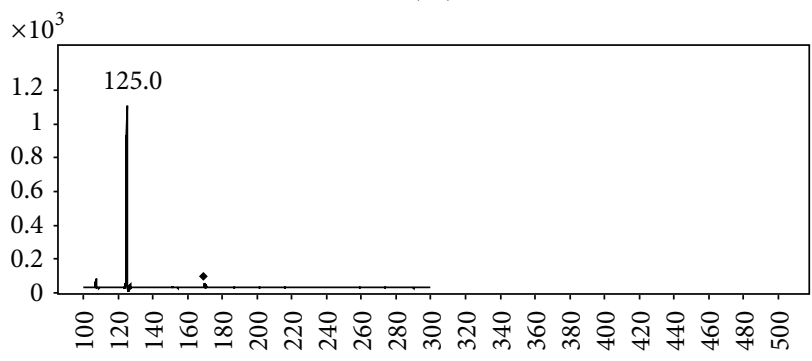

$(m / z)$

(b2)

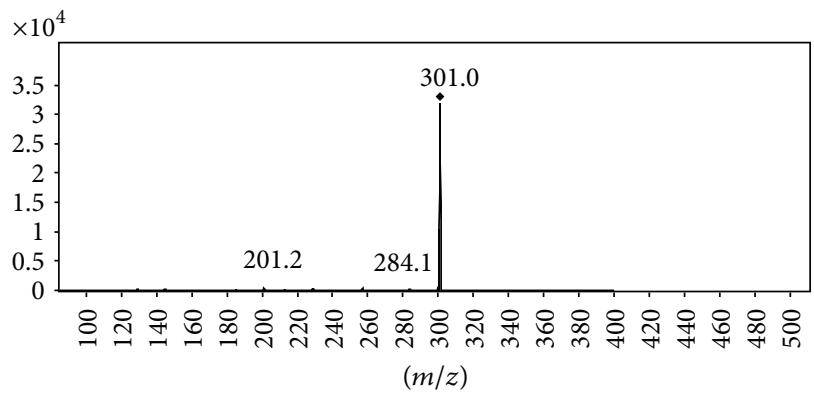

(c2)

(d)

FIGURE 2: Structure and mass spectra of corilagin, GA, and EA and typical LC-MS chromatogram of PTF in HBSS solution. Scan spectra (al, $\mathrm{b} 1$, and $\mathrm{cl}$ ) and product ion (a2, b2, and $\mathrm{c} 2)$ of corilagin, GA, and EA were got in negative ion mode. In the typical LC-MS chromatogram (d), peaks ${ }^{*} 1,{ }^{*} 2$, and ${ }^{*} 3$ correspond to GA, corilagin, and EA in PTF with concentration of $5 \mu \mathrm{g} / \mathrm{mL}$ and the chromatogram was using TIC mode. The diamond shape refers to the precursor ion (M-1) of the product ion.

3.4. Transport of PTF across Caco-2 Cell Monolayers. The Caco-2 cells were incubated at the concentration of $1.00 \mathrm{mg} /$ $\mathrm{mL}$ for a period of time from $30 \mathrm{~min}$ to $180 \mathrm{~min}$. Time courses of the absorption of PTF have been summarized in Figure 3. The transport rates of PTF of different concentration at $180 \mathrm{~min}$ are shown in Figure 4. As shown in Figures 3 and 4 , the transport courses of all three compounds demonstrated that their transport percentages and transport rates were increased in a nonlinear manner with the time and concentration. These results suggested that the three 

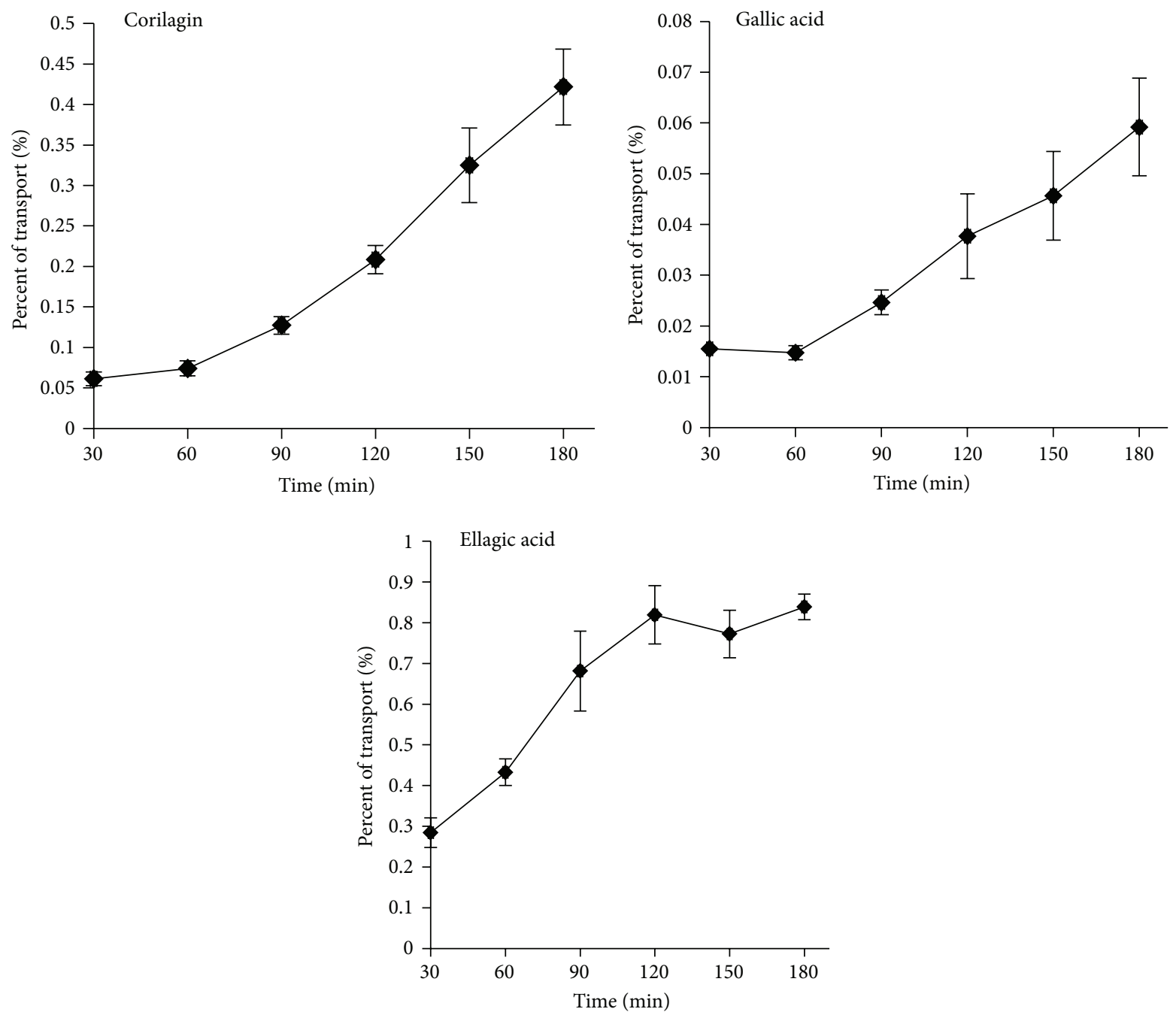

FIgURE 3: The percent transport of the uptake of three compounds in PTF across Caco-2 cell monolayers at different time points during $180 \mathrm{~min}$. Caco-2 cells were incubated with PTF at the concentration of $1.00 \mathrm{mg} / \mathrm{mL}$. Results are expressed as the mean \pm SEM for 3 determinations.

TABLE 1: The intraday and interday precision and recovery of corilagin, GA, and EA in PTF.

\begin{tabular}{|c|c|c|c|c|c|c|}
\hline \multirow{2}{*}{ Chemicals } & \multirow{2}{*}{ Standard samples $(\mathrm{ng} / \mathrm{mL})$} & \multicolumn{2}{|c|}{ Intraday } & \multicolumn{2}{|c|}{ Interday } & \multirow{2}{*}{ Mean recovery $(\%)$} \\
\hline & & Mean $(\mathrm{ng} / \mathrm{mL})$ & RSD (\%) & Mean $(\mathrm{ng} / \mathrm{mL})$ & RSD (\%) & \\
\hline \multirow{3}{*}{ Corilagin } & 32.65 & 39.48 & 3.44 & 38.24 & 4.48 & 117.12 \\
\hline & 163.25 & 156.08 & 2.65 & 153.83 & 4.42 & 94.23 \\
\hline & 326.50 & 308.63 & 3.22 & 301.89 & 4.11 & 92.46 \\
\hline \multirow{3}{*}{ Gallic acid } & 10.08 & 10.44 & 2.55 & 10.06 & 4.77 & 99.80 \\
\hline & 50.40 & 47.25 & 3.26 & 47.05 & 3.37 & 93.35 \\
\hline & 252.00 & 234.92 & 2.06 & 237.83 & 2.99 & 94.38 \\
\hline \multirow{3}{*}{ Ellagic acid } & 98.88 & 117.00 & 2.47 & 117.44 & 2.43 & 118.77 \\
\hline & 494.40 & 519.71 & 1.97 & 503.26 & 4.01 & 101.79 \\
\hline & 988.80 & 952.80 & 2.95 & 930.62 & 4.11 & 94.16 \\
\hline
\end{tabular}

Recovery $(\%)=100 \times($ amount found - original amount $) /$ original amount. 

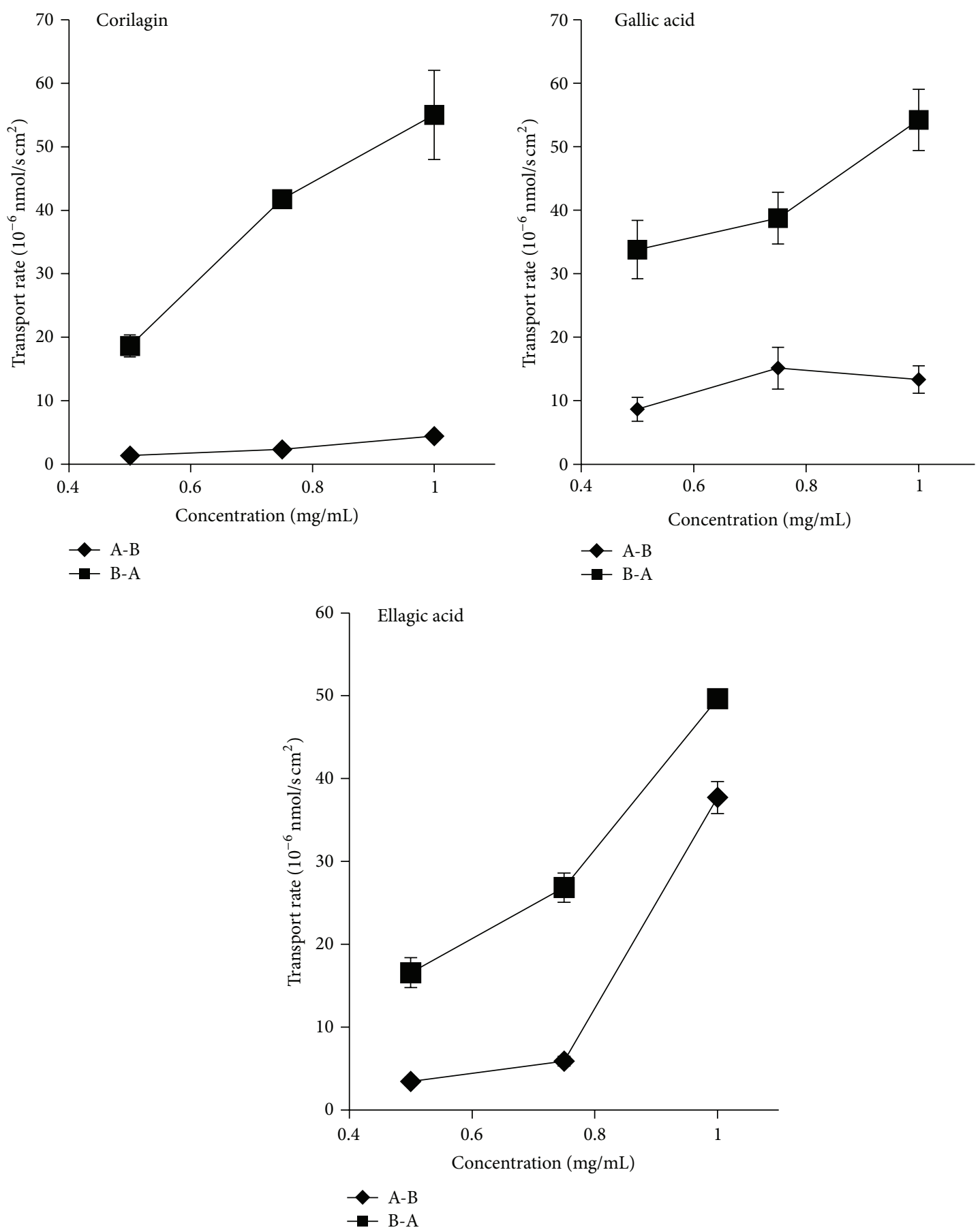

FIGURE 4: The transport rates of the transport of three compounds in PTF across Caco-2 cell monolayers with three concentrations. Caco-2 cells were incubated with PTF for 180 min. Results are expressed as the mean \pm SEM for 3 determinations.

compounds may be transported across the Caco- 2 cell monolayers through protein mediated pathways.

3.5. Vectorial Characteristics of PTF Transport. The $P_{\text {app }}$ values calculated for those constituents of PTF are presented in Table 2. The absorption transport $P_{\text {app }}$ values of corilagin and EA were at the level of $10^{-7} \mathrm{~cm} / \mathrm{s}$, while that of GA was at the level of $10^{-8} \mathrm{~cm} / \mathrm{s}$. The secretion transport $P_{\text {app }}$ values of GA and EA were at the level of $10^{-7} \mathrm{~cm} / \mathrm{s}$, while that of corilagin was at the level of $10^{-6} \mathrm{~cm} / \mathrm{s}$. We found statistically significant differences between absorption and secretion transport values. The PDR values of corilagin and GA were 12.52 and 4.17, respectively. The PDR levels suggested that the permeability of corilagin and GA in PTF was directional and could be affected by the efflux transport inhibitors.

3.6. Influence of Different Inhibitor on the Transport of PTF in the Caco-2 Cell Model. The effects of the transport inhibitors 


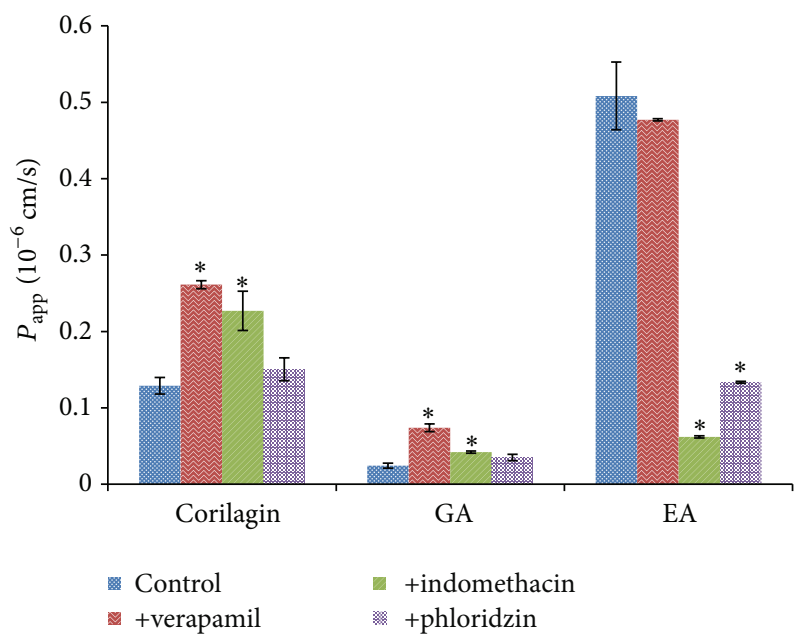

FIGURE 5: The effects of the transporters on the uptake of three compounds in PTF across Caco- 2 cell monolayers. The concentrations of verapamil, indomethacin, and phloridzin were 100,10 , and $50 \mu \mathrm{M}$, respectively. Caco-2 cells were incubated with PTF at the concentration of $1.00 \mathrm{mg} / \mathrm{mL}$ for $120 \mathrm{~min}$. Results are expressed as the mean \pm SEM for 3 determinations. ${ }^{*} P<0.05$ compared with the control group.

TABLE 2: The bidirectional transport of corilagin, GA, and EA in PTF through Caco-2 monolayers.

\begin{tabular}{lccc}
\hline Chemicals & $P_{\text {app AP } \rightarrow \mathrm{BL}} / 10^{-6} \mathrm{~cm} / \mathrm{s}$ & $P_{\text {app BL } \rightarrow \mathrm{AP}} / 10^{-6} \mathrm{~cm} / \mathrm{s}$ & $\mathrm{PDR}$ \\
\hline Corilagin & $0.174 \pm 0.019$ & $2.178 \pm 0.228$ & 12.52 \\
Gallic acid & $0.024 \pm 0.004$ & $0.100 \pm 0.009$ & 4.17 \\
Ellagic acid & $0.347 \pm 0.018$ & $0.456 \pm 0.010$ & 1.31 \\
\hline
\end{tabular}

The $P_{\text {app }}$ values were determined for a period of $180 \mathrm{~min}$ at the concentration of $1.00 \mathrm{mg} / \mathrm{mL}$; results were expressed as the mean $\pm \operatorname{SEM}(n=3)$.

including verapamil, indomethacin, and phloridzin on the absorption of these compounds were showed in Figure 5. The transports of corilagin and GA were significantly increased when they were incubated with verapamil and indomethacin, but no considerable change was observed when they were incubated with phloridzin. The $P_{\text {app }}$ value of EA was strongly decreased in the presence of indomethacin and phloridzin, while no substantial difference was detected in the presence of verapamil. These results indicated that the possible transport pathway of corilagin and GA from PTF may be a P-gp and MRPs mediated route in Caco- 2 cells, and the transport of EA from PTF may be associated with OATP and SGLT1.

\subsection{Epithelial Tight Junction Modulate Effect on PTF Trans-} port. As shown in Figure 6, the $P_{\text {app }}$ values of the three compounds were enhanced 4.20- to 23.83-fold by SDS and PLL. These results suggested that the poor absorption of three compounds of PTF in Caco-2 cell monolayer may be due to the paracellular transport.

\section{Discussion}

Investigations of transport mechanism of herbal extracts across Caco-2 cell monolayer model have been widely

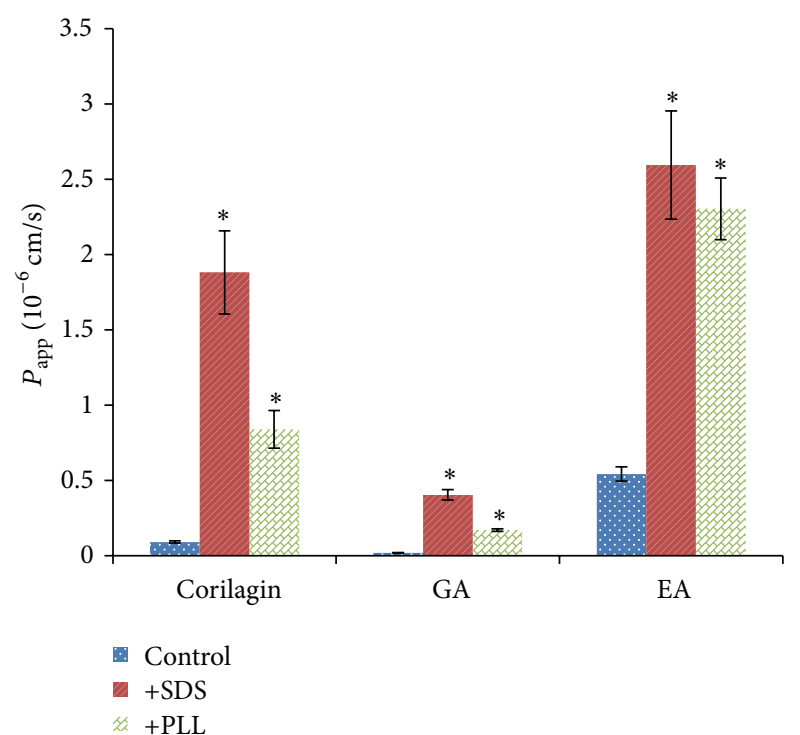

Figure 6: The effects of the tight junction modulators on the uptake of three compounds in PTF across Caco-2 cell monolayers. The concentrations of both SDS and PLL were $50 \mu \mathrm{g} / \mathrm{mL}$. Caco-2 cells were incubated with PTF at the concentration of $1.00 \mathrm{mg} / \mathrm{mL}$ for $120 \mathrm{~min}$. Results are expressed as the mean $\pm \mathrm{SEM}$ for 3 determinations. ${ }^{*} P<0.05$ compared with the control group.

concerned in recent years $[34,35]$. In this study, the model was employed to investigate the intestinal absorption of the hydrolyzable tannin (corilagin) and its hydrolysates (GA and EA) from PTF. A preliminary analysis demonstrated that the concentrations of the three compounds after absorption were so low that they could not be detected by HPLC-UV method. LC-MS method was selected for its high sensitivity and selectivity, which was used in the analysis of transport of herbal extracts across Caco-2 cell monolayer model [36]. After incubation with Caco-2 monolayers for different periods of time from $30 \mathrm{~min}$ to $180 \mathrm{~min}$, the three compounds from PTF appeared hardly absorbable as their $P_{\text {app }}$ values were at the level or even lower than those of sodium fluorescein $[32,33]$. These results were supported by our in vivo experiment and the prior reports: the absorption of EA was enormously poor after oral administration, and the $P_{\text {app }}$ value of GA across Caco-2 cell monolayer was about $0.20 \times 10^{-6} \mathrm{~cm} / \mathrm{s}[37,38]$.

The mechanism of the poor absorption of PTF was further investigated. According to Liu's report, the nonlinear time course of orientin and vitexin may be explained by the saturation of transporters [22]. The similar time course characters of corilagin and GA showed an uptrend of transport with incubation time increase, which demonstrated the contribution of the efflux transporters (Figure 3). In addition, the time course of EA reached a plateau with incubation time at $2 \mathrm{~h}$, which indicated the participation of absorption transporters (Figure 3). When PDR was more than 1.5, the transport was considered to be directional [39]. The PDR levels of GA and corilagin were 4.17 and 12.52, respectively, which suggested the participation of efflux transport protein. 
The P-gp, MRPs, and BCRP are efflux transporters; OATP, SGLT1, and MCT are uptake proteins. In this study, the transport of corilagin and GA involved P-gp and MRPs mediated active efflux in addition to passive diffusion, which was evidenced by their $P_{\text {app }}$ values (less than $10^{-6} \mathrm{~cm} / \mathrm{s}$ ), PDR levels (more than 1.5) (Table 2), the nonlinear transport with time and concentration (Figures 3-4), and the significantly increased transport in the presence of verapamil and indomethacin. The transport of EA may be a little different, the PDR level was less than 1.5, and the absorption was obviously decreased in the presence of indomethacin and phloridzin, which demonstrated that EA was absorbed mainly through passive diffusion in combination with OATP and SGLT1 mediated active uptake. The transport of polyphenols was proved by previous reports to be mediated by both efflux and uptake proteins. The transport of resveratrol, apigenin, and puerarin was mediated by P-gp and MRPs $[21,35]$; the uptake of chrysophanol and emodin was with the participation of SGLT1 [21]; the absorption of forsythoside A was participated by OATP [26]. The inhibitors of P-gp and MRPs but not SGLT1 or OATP may benefit the absorption of $\mathrm{PTF}$ in vivo.

The transepithelial transport of GA was reported earlier [38], and its $P_{\text {app }}$ was about $0.20 \times 10^{-6} \mathrm{~cm} / \mathrm{s}$, which was much higher than that of GA in PTF $\left(0.024 \times 10^{-6} \mathrm{~cm} / \mathrm{s}\right)$. The absorption between pure compounds and that contented in herbal extracts appeared different, which was well proved in previous findings. As reported in the literature, the presence of other compounds in Aconitum carmichaeli [40] and Schisandra chinensis [41] extracts assisted the transport of aconitine and gomisin A, respectively. Besides, the components of apple peel extracts hindered the absorption of quercetin across the Caco-2 cells [34]. The interactions of other components in PTF on the absorption of GA should be considered in the next step.

Medium chain fatty acids (MCFAs) and polycations can enhance the absorption by affecting the tight junction permeability of Caco-2 cells [29, 30, 42]. SDS and PLL are typical MCFA and polycation, respectively. Both SDS and PLL were shown to affect intestinal epithelial monolayers differently in different time and dose. In our study, we tested the incubation time and dose and found that, before incubation with SDS $(50 \mu \mathrm{g} / \mathrm{mL})$ and PLL $(50 \mu \mathrm{g} / \mathrm{mL})$ for $30 \mathrm{~min}$, the TEER values of monolayers were lower than $500 \Omega / \mathrm{cm}^{2}\left(250-350 \Omega / \mathrm{cm}^{2}\right)$; after removing the SDS and PLL the TEER values could rise to $500-600 \Omega / \mathrm{cm}^{2}$ in two hours. Both SDS and PLL greatly improved the transport of corilagin, GA, and EA. Other tight junction modulators such as EDTA and sodium caprate could enhance the transport of 3,6'-disinapoylsucrose [28]. These results further suggested that the paracellular transport appeared to be main limiting factor for the uptake of three compounds in Caco-2 cell monolayers.

\section{Conclusion}

This study indicated that the transports of the corilagin and its hydrolysates GA and EA from PTF were via passive diffusion combined with protein mediated transport. Specifically, the absorption of corilagin and GA was P-gp and MRPs mediated efflux, and the active uptake of EA was associated with the OATP and SGLT1 protein. The improvement of the transport of these compounds in PTF caused by transport protein inhibitors or epithelial tight junction modulators may be helpful for the increasing of oral absorption and improving of antitumor effect.

\section{Competing Interests}

The authors declare that they have no competing interests.

\section{Acknowledgments}

This project was supported by the National Natural Science Foundation of China (81274187 and 81274006).

\section{References}

[1] T. Yoshida, Y. Amakura, and M. Yoshimura, "Structural features and biological properties of ellagitannins in some plant families of the order myrtales," International Journal of Molecular Sciences, vol. 11, no. 1, pp. 79-106, 2010.

[2] L. Jia, H. Jin, M. Lv et al., "Abstract 5550: corilagin, an antitumor herb medicine, sensitizes paclitaxel and carboplatin by primarily inhibiting glycolysis pathways in epithelial ovarian cancer," Cancer Research, vol. 75, no. 15, supplement, pp. 55505550, 2015.

[3] L. Jia, H. Jin, J. Zhou et al., "A potential anti-tumor herbal medicine, Corilagin, inhibits ovarian cancer cell growth through blocking the TGF- $\beta$ signaling pathways," BMC Complementary and Alternative Medicine, vol. 13, article 33, 2013.

[4] Y.-J. Guo, T. Luo, F. Wu et al., "Corilagin protects against HSV1 encephalitis through inhibiting the TLR2 signaling pathways in vivo and in vitro," Molecular Neurobiology, vol. 52, no. 3, pp. 1547-1560, 2014.

[5] J. Moreira, L. C. Klein-Júnior, V. C. Filho, and F. D. C. Buzzi, "Anti-hyperalgesic activity of corilagin, a tannin isolated from Phyllanthus niruri L. (Euphorbiaceae)," Journal of Ethnopharmacology, vol. 146, no. 1, pp. 318-323, 2013.

[6] M. Shimizu, S. Shiota, T. Mizushima et al., "Marked potentiation of activity of $\beta$-lactams against methicillin-resistant Staphylococcus aureus by corilagin," Antimicrobial Agents \& Chemotherapy, vol. 45, no. 11, pp. 3198-3201, 2001.

[7] B. Badhani, N. Sharma, and R. Kakkar, "Gallic acid: a versatile antioxidant with promising therapeutic and industrial applications," RSC Advances, vol. 5, no. 35, pp. 27540-27557, 2015.

[8] A. S. Meyer, M. Heinonen, and E. N. Frankel, "Antioxidant interactions of catechin, cyanidin, caffeic acid, quercetin, and ellagic acid on human LDL oxidation," Food Chemistry, vol. 61, no. 1-2, pp. 71-75, 1998.

[9] W. H. Park and S. H. Kim, "MAPK inhibitors augment gallic acid-induced A549 lung cancer cell death through the enhancement of glutathione depletion," Oncology Reports, vol. 30, no. 1, pp. 513-519, 2013.

[10] R. Vicinanza, Y. Zhang, S. M. Henning, and D. Heber, "Pomegranate juice metabolites, ellagic acid and urolithin A, synergistically inhibit androgen-independent prostate cancer cell growth via distinct effects on cell cycle control and apoptosis," Evidence-Based Complementary and Alternative Medicine, vol. 2013, Article ID 247504, 12 pages, 2013. 
[11] Q. Xia, P. Xiao, L. Wang, and J. Kong, "Ethnopharmacology of Phyllanthus emblica L.," Zhongguo Zhong Yao Za Zhi, vol. 22, no. 9, pp. 515-525, 1998 (Chinese).

[12] L.-Z. Zhang, W.-H. Zhao, Y.-J. Guo, G.-Z. Tu, S. Lin, and L.G. Xin, "Studies on chemical constituents in fruits of tibetan medicine Phyllanthus emblica," Zhongguo Zhongyao Zazhi, vol. 28, no. 10, pp. 942-943, 2003.

[13] H.-J. Zhao, T. Liu, X. Mao et al., "Fructus Phyllanthi tannin fraction induces apoptosis and inhibits migration and invasion of human lung squamous carcinoma cells in vitro via MAPK/MMP pathways," Acta Pharmacologica Sinica, vol. 36, no. 6, pp. 758-768, 2015.

[14] L. F. Wu, Pharmacokinetic Study of Fructus Phyllanthi tannin Fraction, Beijing University of Chinese Medicine, Beijing, China, 2014 (Chinese).

[15] M. Pinto, S. Robine-Leon, and M. D. Appay, "Enterocyte-like differentiation and polarization of the human colon carcinoma cell line Caco-2 in culture," Biology of the Cell, vol. 47, no. 3, pp. 323-330, 1983.

[16] A. R. Hilgers, R. A. Conradi, and P. S. Burton, "Caco-2 cell monolayers as a model for drug transport across the intestinal mucosa," Pharmaceutical Research, vol. 7, no. 9, pp. 902-910, 1990.

[17] H. Gutmann, G. Fricker, M. Török, S. Michael, C. Beglinger, and J. Drewe, "Evidence for different ABC transporters in Caco-2 cells modulating drug uptake," Pharmaceutical Research, vol. 16, no. 3, pp. 402-407, 1999.

[18] T. Hirohashi, H. Suzuki, X.-Y. Chu, I. Tamai, A. Tsuji, and Y. Sugiyama, "Function and expression of multidrug resistanceassociated protein family in human colon adenocarcinoma cells (Caco-2)," Journal of Pharmacology and Experimental Therapeutics, vol. 292, no. 1, pp. 265-270, 2000.

[19] G. Englund, F. Rorsman, A. Rönnblom et al., "Regional levels of drug transporters along the human intestinal tract: coexpression of ABC and SLC transporters and comparison with Caco-2 cells," European Journal of Pharmaceutical Sciences, vol. 29, no. 3-4, supplement, pp. 269-277, 2006.

[20] J. I. Fletcher, M. Haber, M. J. Henderson, and M. D. Norris, "ABC transporters in cancer: more than just drug efflux pumps," Nature Reviews Cancer, vol. 10, no. 2, pp. 147-156, 2010.

[21] Z. Teng, C. Yuan, F. Zhang et al., "Intestinal absorption and first-pass metabolism of polyphenol compounds in rat and their transport dynamics in caco-2 cells," PLoS ONE, vol. 7, no. 1, Article ID e29647, 2012.

[22] L. Liu, L. Guo, C. Zhao, X. Wu, R. Wang, and C. Liu, "Characterization of the intestinal absorption of seven flavonoids from the flowers of Trollius chinensis using the Caco-2 cell monolayer model," PLoS ONE, vol. 10, no. 3, Article ID e0119263, 2015.

[23] J. B. Vaidyanathan and T. Walle, "Cellular uptake and efflux of the tea flavonoid (-)epicatechin-3-gallate in the human intestinal cell line Caco-2," Journal of Pharmacology \& Experimental Therapeutics, vol. 307, no. 2, pp. 745-752, 2003.

[24] M. P. Draper, R. L. Martell, and S. B. Levy, "Indomethacinmediated reversal of multidrug resistance and drug efflux in human and murine cell lines overexpressing MRP, but not Pglycoprotein," British Journal of Cancer, vol. 75, no. 6, pp. 810815, 1997.

[25] H. M. Prime-Chapman, R. A. Fearn, A. E. Cooper, V. Moore, and B. H. Hirst, "Differential multidrug resistance-associated protein 1 through 6 isoform expression and function in human intestinal epithelial Caco-2 cells," Journal of Pharmacology \& Experimental Therapeutics, vol. 311, no. 2, pp. 476-484, 2004.
[26] W. Zhou, L.-Q. Di, J. Wang et al., "Intestinal absorption of forsythoside A in in situ single-pass intestinal perfusion and in vitro Caco-2 cell models," Acta Pharmacologica Sinica, vol. 33, no. 8, pp. 1069-1079, 2012.

[27] G. Burckhardt and B. C. Burckhardt, "In vitro and in vivo evidence of the importance of organic anion transporters (OATs) in drug therapy," in Drug Transporters, pp. 29-104, Springer, Heidelberg, Germany, 2011.

[28] Y. Chen, X. Liu, R. Pan et al., "Intestinal transport of 3,6'disinapoylsucrose, a major active component of Polygala tenuifolia, using Caco-2 cell monolayer and in situ rat intestinal perfusion models," Planta Medica, vol. 79, no. 15, pp. 1434-1439, 2013.

[29] E. K. Anderberg and P. Artursson, "Epithelial transport of drugs in cell culture. VIII: effects of sodium dodecyl sulfate on cell membrane and tight junction permeability in human intestinal epithelial (Caco-2) cells," Journal of Pharmaceutical Sciences, vol. 82, no. 4, pp. 392-398, 1993.

[30] G. Ranaldi, I. Marigliano, I. Vespignani, G. Perozzi, and Y. Sambuy, "The effect of chitosan and other polycations on tight junction permeability in the human intestinal Caco-2 cell line," Journal of Nutritional Biochemistry, vol. 13, no. 3, pp. 157-167, 2002.

[31] X.-F. Sun, H.-Y. Zhang, Q. Xia et al., "HPLC-fingerprint-based quality evaluation on a Tibetan medicine Phyllanthus emblica and its tannin parts," Zhongguo Zhongyao Zazhi, vol. 39, no. 7, pp. 1173-1178, 2014.

[32] Y. Konishi, K. Hagiwara, and M. Shimizu, "Transepithelial transport of fluorescein in Caco-2 cell monolayers and use of such transport in in vitro evaluation of phenolic acid availability," Bioscience, Biotechnology \& Biochemistry, vol. 66, no. 11, pp. 2449-2457, 2002.

[33] G. Sandri, M. C. Bonferoni, S. Rossi et al., "Nanoparticles based on N-trimethylchitosan: evaluation of absorption properties using in vitro (Caco-2 cells) and ex vivo (excised rat jejunum) models," European Journal of Pharmaceutics and Biopharmaceutics, vol. 65, no. 1, pp. 68-77, 2007.

[34] J. Boyer, D. Brown, and R. H. Liu, "Uptake of quercetin and quercetin 3-glucoside from whole onion and apple peel extracts by Caco-2 cell monolayers," Journal of Agricultural \& Food Chemistry, vol. 52, no. 23, pp. 7172-7179, 2004.

[35] X.-L. Liang, L.-J. Zhao, Z.-G. Liao et al., “Transport properties of puerarin and effect of Radix Angelicae Dahuricae extract on the transport of puerarin in Caco-2 cell model," Journal of Ethnopharmacology, vol. 144, no. 3, pp. 677-682, 2012.

[36] K. Y.-Z. Zheng, R. C.-Y. Choi, A. J.-Y. Guo et al., “The membrane permeability of Astragali Radix-derived formononetin and calycosin is increased by Angelicae Sinensis Radix in Caco-2 cells: a synergistic action of an ancient herbal decoction Danggui Buxue Tang," Journal of Pharmaceutical and Biomedical Analysis, vol. 70, pp. 671-679, 2012.

[37] L. Yan, P. Yin, C. Ma, and Y. Liu, "Method development and validation for pharmacokinetic and tissue distributions of ellagic acid using ultrahigh performance liquid chromatographytandem mass spectrometry (UPLC-MS/MS)," Molecules, vol. 19, no. 11, pp. 18923-18935, 2014.

[38] Y. Konishi, S. Kobayashi, and M. Shimizu, “Transepithelial transport of p-coumaric acid and gallic acid in Caco-2 cell monolayers," Bioscience, Biotechnology and Biochemistry, vol. 67, no. 11, pp. 2317-2324, 2003.

[39] K. Gao, J. Sun, and Z. G. He, "Important application of Caco2 cell monolayers in the study of the intestinal oral absorption," 
Journal of Shenyang Pharmaceutical University, vol. 22, no. 6, pp. 469-474, 2005 (Chinese).

[40] N. Li, R. Tsao, Z. Sui, J. Ma, Z. Liu, and Z. Liu, "Intestinal transport of pure diester-type alkaloids from an aconite extract across the Caco-2 cell monolayer model," Planta Medica, vol. 78, no. 7, pp. 692-697, 2012.

[41] V. L. M. Madgula, B. Avula, Y. W. Choi et al., "Transport of Schisandra chinensis extract and its biologically-active constituents across Caco-2 cell monolayers-an in-vitro model of intestinal transport," Journal of Pharmacy \& Pharmacology, vol. 60, no. 3, pp. 363-370, 2008.

[42] T. Lindmark, Y. Kimura, and P. Artursson, "Absorption enhancement through intracellular regulation of tight junction permeability by medium chain fatty acids in Caco-2 cells," Journal of Pharmacology and Experimental Therapeutics, vol. 284, no. 1, pp. 362-369, 1998. 


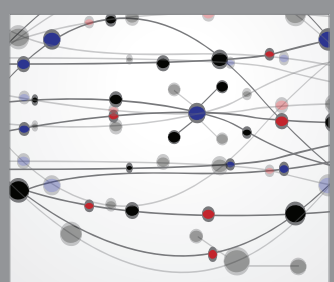

The Scientific World Journal
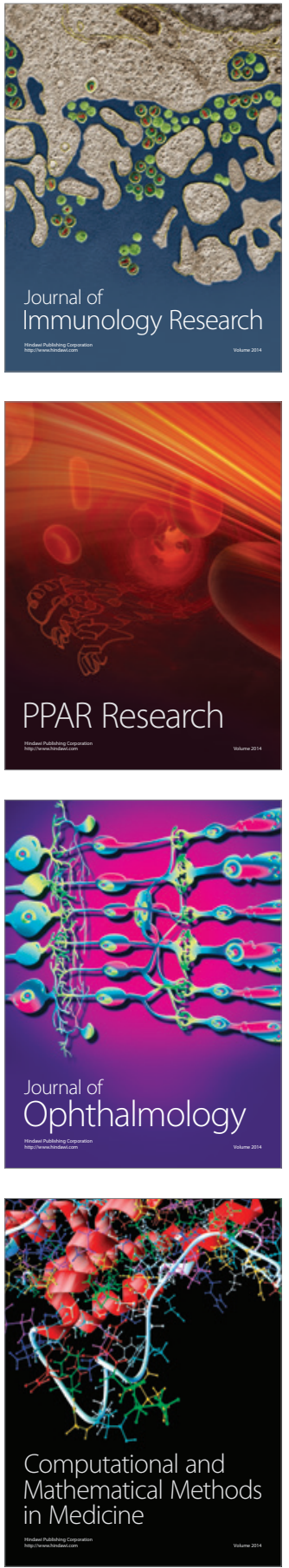

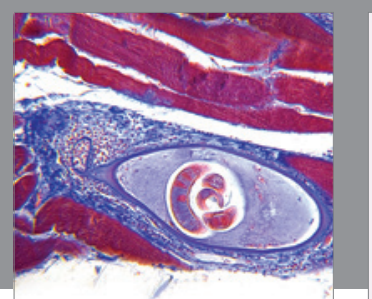

Gastroenterology Research and Practice

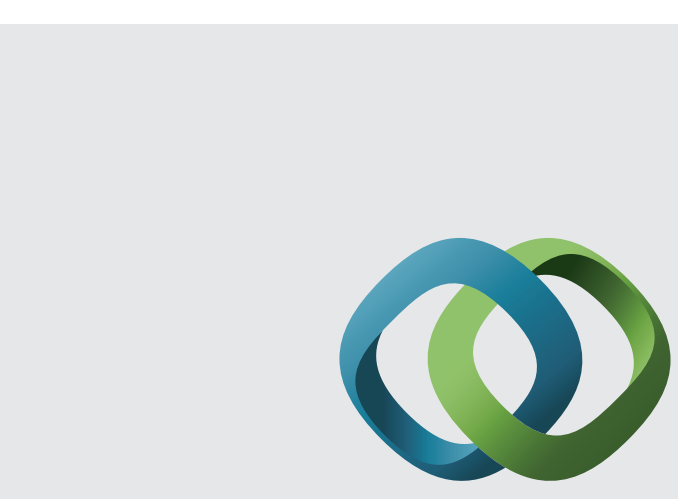

\section{Hindawi}

Submit your manuscripts at

http://www.hindawi.com
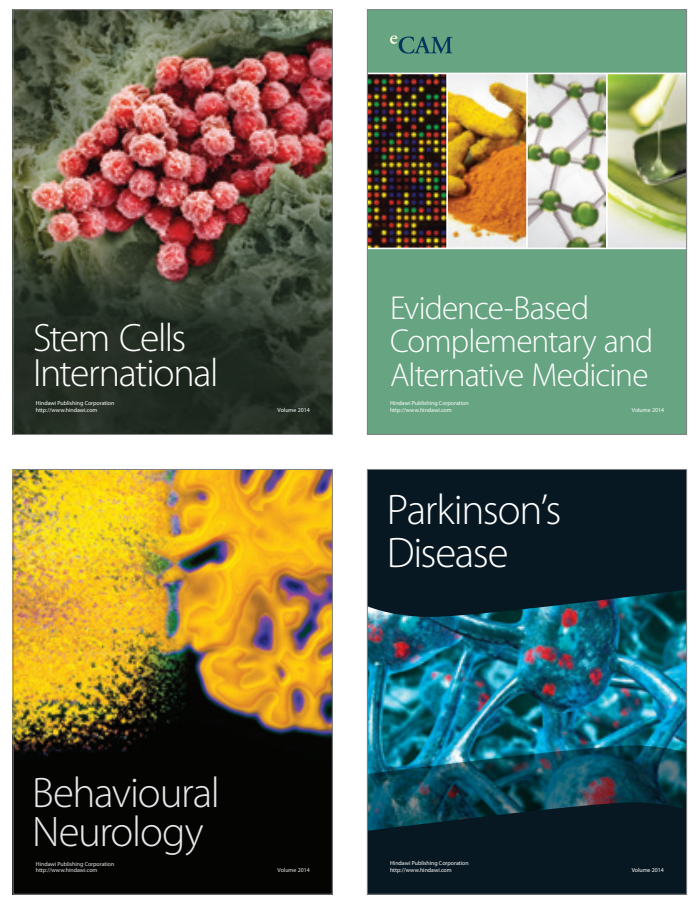
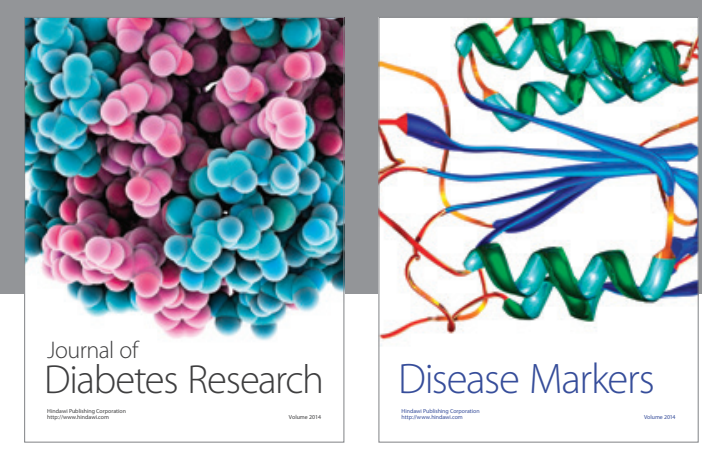

Disease Markers
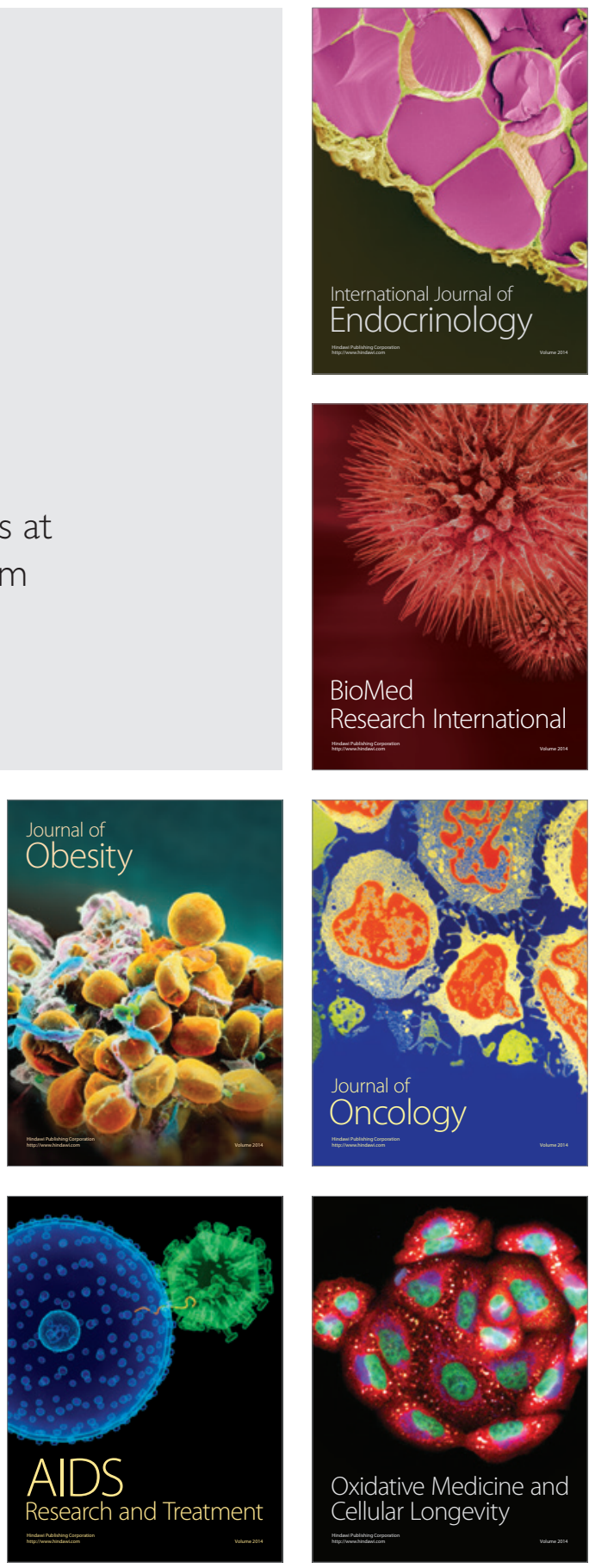